\title{
BMJ Open Patient engagement in hospital health service planning and improvement: a scoping review
}

\author{
Laurel Liang, ${ }^{1}$ Albina Cako, ${ }^{1}$ Robin Urquhart, ${ }^{2}$ Sharon E Straus, ${ }^{3}$ Walter P Wodchis, ${ }^{4}$ \\ G. Ross Baker, ${ }^{4}$ Anna R Gagliardi ${ }^{1}$
}

To cite: Liang L, Cako A, Urquhart $\mathrm{R}$, et al. Patient engagement in hospital health service planning and improvement: a scoping review. BMJ Open 2018;8:e018263. doi:10.1136/ bmjopen-2017-018263

- Prepublication history and additional material for this paper are available online. To view these files, please visit the journal (http://dx.doi.org/10. 1136/bmjopen-2017-018263).

Received 17 June 2017 Revised 18 December 2017 Accepted 19 December 2017

Check for updates

${ }^{1}$ Toronto General Hospital Research Institute, University Health Network, Toronto, Ontario, Canada

${ }^{2}$ Beatrice Hunter Cancer Research Institute, Dalhousie University, Halifax, UK

${ }^{3}$ Department of Medicine, University of Toronto, Toronto, Ontario, Canada

${ }^{4}$ Institute of Health Policy, Management and Evaluation, University of Toronto, Toronto, Ontario, Canada

Correspondence to

Anna R Gagliardi;

anna.gagliardi@uhnresearch.ca

\section{ABSTRACT}

Objectives Patient engagement (PE) improves patient, organisation and health system outcomes, but most research is based on primary care. The primary purpose of this study was to describe the characteristics of published empirical research that evaluated PE in hospital health service improvement.

Design Scoping review.

Methods Five databases were searched from 2006 to September 2016. English language studies that evaluated patient or provider beliefs, participation in PE, influencing factors or impact were eligible. Screening and data extraction were done in triplicate. PE characteristics, influencing factors and impact were extracted and summarised.

Results From a total of 3939 search results, 227 studies emerged as potentially relevant; of these, 217 were not eligible, and 10 studies were included in the review. None evaluated behavioural interventions to promote or support PE. While most studies examined involvement in standing committees or projects, patient input and influence on decisions were minimal. Lack of skill and negative beliefs among providers were PE barriers. PE facilitators included careful selection and joint training of patients and providers, formalising patient roles, informal interaction to build trust, involving patients early in projects, small team size, frequent meetings, active solicitation of patient input in meetings and debriefing after meetings. Asking patients to provide insight into problems rather than solutions and deploying provider champions may enhance patient influence on hospital services.

Conclusions Given the important role of PE in improving hospital services and the paucity of research on this topic, future research should develop and evaluate behavioural interventions for PE directed at patients and providers informed by the PE barriers and facilitators identified here. Future studies should also assess the impact on various individual and organisational outcomes.

\section{BACKGROUND}

Patient engagement (PE) in healthcare is a worldwide priority because evidence shows that it improves numerous patient outcomes, such as satisfaction with care received, and health system outcomes such as cost-effective service delivery. ${ }^{12}$ Research has largely focused on engaging patients in securing their own

\section{Strengths and limitations of this study}

- By describing the characteristics of published empirical research that evaluated patient engagement $(\mathrm{PE})$ in hospital health service improvement, this research identified what is known about how to achieve PE in hospital health service improvement including facilitators and barriers.

- This scoping review also exposed gaps in knowledge that inform future research in this area: develop and evaluate different types of $\mathrm{PE}$ along the continuum of consultation, involvement and partnership; develop behavioural interventions targeted at patients and providers to support PE; and evaluate the impact of PE on clinical outcomes.

- This study used rigorous scoping review methods including a detailed search of multiple databases that complied with standards for search strategies, employed a framework of patient and family engagement to characterise PE activities, and complied with standards for the conduct and reporting of reviews.

- All relevant studies may not have been identified or included because the search strategy may not have been sufficiently comprehensive. Grey literature was not explored, non-English studies were excluded and the screening criteria may have been overly stringent.

- Few studies were eligible and those studies provided few specific details about what patients were meant to do or actually did (eg, mode or frequency of engagement, information they contributed or how it was used). Thus, little knowledge was revealed about how to optimise PE in hospital service planning and improvement.

appropriate, effective, safe and responsive healthcare, also referred to as patient-centred care. ${ }^{3} 4$ Previous syntheses of research on PE in their own care generated insight on approaches or technologies to support PE. ${ }^{56}$ Examples include providing care in a compassionate and empowering manner by sharing information and being sensitive to patient needs, ${ }^{7}$ interprofessional collaboration and case management to optimise the 
coordination of care, and prompting patients to enhance the safety of their own care by reminding healthcare professionals to use practices such as hand hygiene or the surgical safety checklist. ${ }^{8}$

Patients can also be engaged in designing or improving health services through activities such as completing surveys about their care experiences, or serving as advisors or members of governance or quality improvement committees. ${ }^{9}$ Research, largely in outpatient settings, has identified strategies that support PE in service improvement; for example, joint training of patients and frontline healthcare professionals, clearly defined roles and responsibilities, and trained facilitators to coach teams. ${ }^{10}$ However, such ideal environments may not be the norm. A systematic review of 26 studies published from 2000 to 2015 found that barriers of PE in health service improvement were lack of training and uncertainty about role among patients, and healthcare professional uncertainty about how to work with patients and act on their feedback, resulting in token PE. ${ }^{11}$ Similarly, a qualitative systematic review of 11 studies published from 2003 to 2012 found that PE in service improvement was challenged by inadequate planning and differing patient and healthcare professional views, and that PE was token in most circumstances and did not lead to improvements. ${ }^{12}$ Therefore, information is needed on the effective strategies and optimal conditions for PE in service improvement. Such knowledge could provide insight into how to support widespread implementation of PE and greater translation of the patient voice to improved services.

Research on PE in service improvement has thus far largely focused on the primary care setting. ${ }^{10-12}$ A considerable proportion of healthcare is delivered in hospitals, which also seeks to optimise service delivery and associated outcomes through PE. ${ }^{13}$ Hospital care differs from primary care in setting, conditions/diseases, procedures and providers; therefore, PE activities, supportive conditions and outcomes may also differ. The quantity and nature of research on PE in hospital-based service improvement have not been previously characterised. The primary purpose of this study was to describe the characteristics of published empirical research that evaluated PE in hospital health service improvement. In so doing, we also sought to describe the types, extent, determinants, interventions and impact of service improvement PE in the hospital context.

\section{METHODS}

\section{Approach}

Rather than a traditional systematic review that seeks to describe outcomes, a scoping review was conducted. ${ }^{14} 15$ This approach was employed to acquire an understanding of the extent, range and nature of research on this topic, describe PE for hospital service improvement and its determinants and impact, and identify issues that warrant further research. A scoping review involves five steps: scoping, searching, screening, data extraction and data analysis. The Preferred Reporting Items for Systematic Reviews and Meta-Analyses criteria guided the conduct and reporting of this review. ${ }^{16}$ Data were publicly available, so institutional review board approval was not necessary. A protocol for this review was not registered.

\section{Scoping}

The scoping step involved becoming familiar with the literature on this topic. A preliminary search was conducted in MEDLINE using Medical Subject Headings including, but not limited to, consumer participation or patient participation and (programme evaluation or quality improvement or hospital administration or hospital planning or health services research. ARG and two trainees (LL, AC) screened titles and abstracts of the search results, which informed the ultimate search strategy and were used to generate eligibility criteria based on the Population, Intervention, Comparisons and Outcomes (PICO) framework. All members of the research team reviewed eligibility criteria and provided feedback, which was used to refine the eligibility criteria. The research team included a physician with expertise in geriatric medicine (SES); health services researchers with expertise in patient safety, quality improvement and PE (WPW, RB, SES, RU, ARG); and experts in evidence syntheses (ARG, RU, SES).

\section{Eligibility criteria}

Population referred to both patients and providers. Patients included adults (aged 18+years) who visited hospitals in any ambulatory, emergent or inpatient capacity for any healthcare issue, condition or disease; or were family members, care givers or well members of the public (all henceforth referred to as patients). Providers were included because research shows that PE is more likely when providers are trained for, and also engaged in PE. ${ }^{10-12}$ Providers included any type of clinicians, executives or managers working in hospitals of any type. Interventions included consulting about or engaging patients or providers in hospital health service improvement activities of any type including governance, service planning, delivery, evaluation or quality improvement, or research to inform service design or improvement. ${ }^{9}$ Interventions also included strategies directed at either patients or providers to promote or support PE in service improvement. With respect to comparisons, studies were eligible if they explored the ways that patients (or family members/ caregivers/public) or providers were involved in PE for hospital service improvement; their views, experiences of, and suggestions to support PE; or evaluated whether and how PE-informed improvements were implemented, strategies used to support PE, or the impact of PE on health services or patient outcomes. Such studies may have included patients or providers with and without exposure to interventions, or before or after exposure to interventions, or receiving different types of interventions. Outcomes were any reported by eligible studies and included, but were not limited to, awareness, knowledge, communication, experiences or impact of PE on hospital 
service improvement, types of PE activities, factors influencing any of these functions, the impact of behavioural interventions to support PE, or the impact of PE. Eligible study designs included English language qualitative (interviews, focus groups, qualitative case studies), quantitative (questionnaires, randomised controlled trials, time series, before/after studies, prospective or retrospective cohort studies, case control studies, economic analyses) or mixed methods studies published in peer-reviewed journals.

Studies were not eligible if they involved providers not based in hospitals or more than half were trainees such as interns, residents or fellows; assessed PE in their own care or PE in health service improvement not based in hospitals; or reported evaluations of patient satisfaction or experience with clinical care. Studies involving the engagement of children and youth, thereby necessitating the involvement of parents or surrogates, were not included as PE processes would differ from those required for adults. While important, given the differing scenario and processes, engaging children and youth in $\mathrm{PE}$ is beyond the scope of this review and best addressed in a separate review. The following publication types were not eligible: systematic reviews, protocols, editorials, commentaries, letters, news items, or meeting abstracts or proceedings. If more than one publication described a single study and reported different data, they were all included but counted as a single study; if they reported the same data, only the most recent publication was included.

\section{Searching}

The search strategy (online supplementary file 1) was developed by ARG (trained as a medical librarian) in conjunction with a professional medial librarian and complied with the Peer Review of Electronic Search Strategies reporting guidelines. ${ }^{17}$ MEDLINE, EMBASE, CINAHL, HealthStar and the Cochrane Library were searched on 20 September 2016 for articles published from 2006 to 2016. The year 2006 was chosen to capture the most recent 10 years of research given that PE for hospital service improvement is a relatively new phenomenon. Systematic reviews were not eligible, but their references and those of all eligible studies were screened to identify additional eligible primary studies. We did not search grey literature because it is time consuming and costly with low yield, and not feasible given that we had few resources for this study; there are no standard methods for doing so, and grey information may be at high risk of bias. ${ }^{18} 19$

\section{Screening}

To prepare for screening of titles and abstracts, ARG, LL and AC independently screened titles and abstracts of the first 25 search results, then discussed discrepancies, and how to interpret and apply the eligibility criteria. LL and AC independently screened titles and abstracts according to specified PICO-based eligibility criteria. All items selected by at least one reviewer were retrieved. Full-text articles were independently screened by LL and AC prior to data extraction; they consulted ARG weekly to resolve uncertainty in inclusion decisions.

\section{Data extraction}

A data extraction form was developed to collect information on author, publication year, country, research design, number and type of participants, description of $\mathrm{PE}$ and/or other interventions, and findings. LL, AC and ARG independently pilot tested data extraction on three articles and compared findings by discussion to refine the data extraction form. AC and LL independently extracted data from eligible articles; all extracted data were independently checked by ARG.

\section{Data analysis}

Summary statistics were used to describe the number and/or proportion of studies by publication year, country, research design, and those that assessed types and extent of PE for hospital service improvement, factors influencing PE, and PE interventions and impact. The types and extent of $\mathrm{PE}$ were categorised independently during data extraction using the Carman and Workman ${ }^{9}$ framework of patient and family engagement that characterises PE by level of engagement (own care, organisational design and governance, policy-making) and continuum of engagement (consultation, involvement, partnership and shared leadership). For this study, which focused on PE for the organisational design and governance of hospitals, types of PE were organised according to activities that consulted, involved or partnered with patients. The quality of individual studies was not assessed because that is not customary for a scoping review. All coauthors reviewed the summary of findings, and their feedback was incorporated in the final version.

\section{RESULTS}

\section{Search results}

From a total of 3939 unique search results, 2227 full-text studies emerged as potentially relevant; of these, $217 \mathrm{did}$ not meet eligibility criteria, and 10 studies were included in the review (figure 1). Data extracted from included studies are summarised in online supplementary file $2 .^{20-29}$

\section{Study characteristics}

Eight of 10 studies reported the number of participating patients (range 10-20) and providers (range 18-142). Another study included 126 participants but did not report the number of patients and providers, ${ }^{22}$ and another study did not report the total number of participants. ${ }^{28}$ One study was published in 2008 and one in 2009, and two studies per year were published in 2010, 2011, 2013 and 2014. Four studies were conducted in the UK (25.0\%), two in Australia (16.7\%) and one each $(8.3 \%)$ in Canada, Norway, Taiwan and Uganda. 


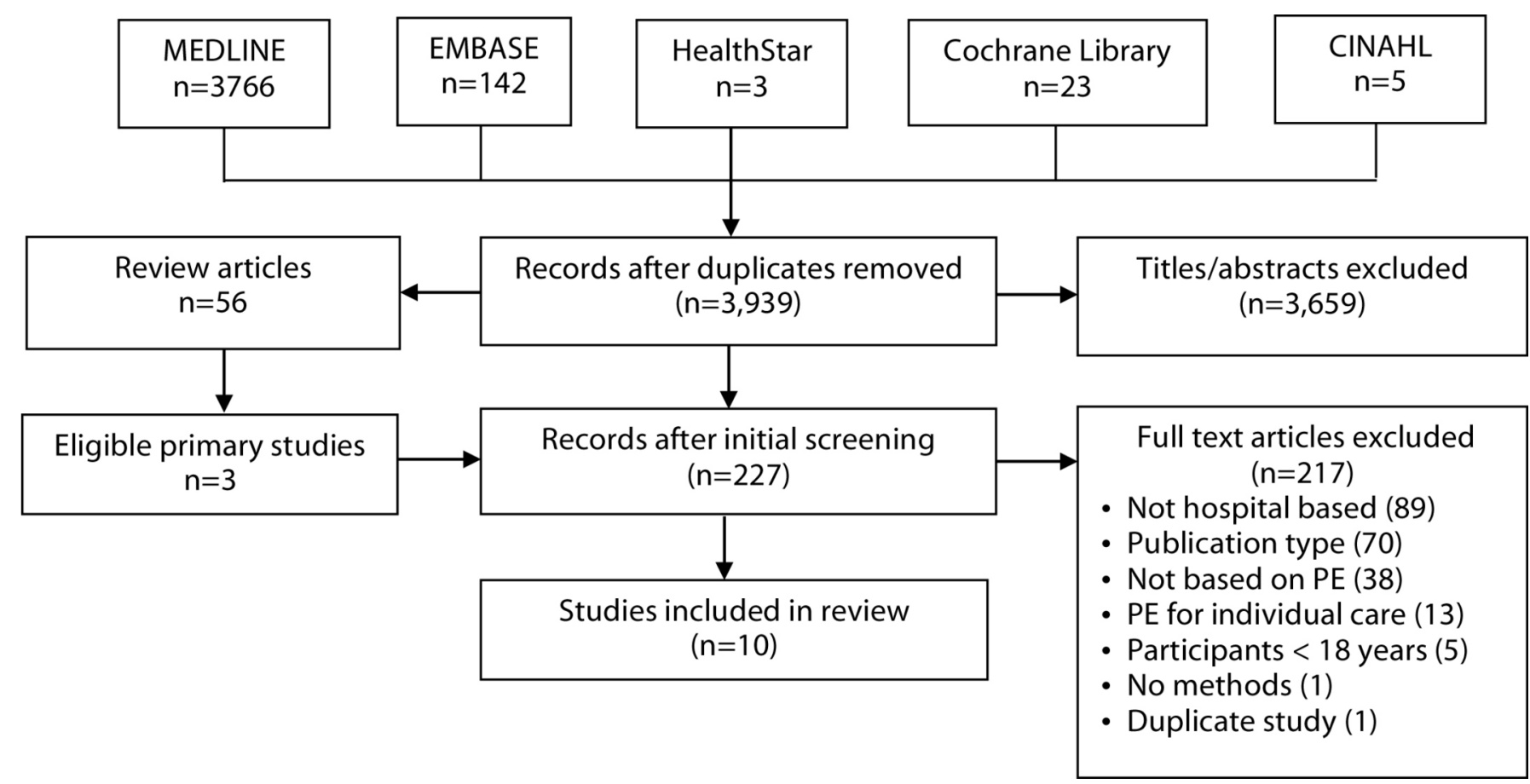

Figure 1 Preferred Reporting Items for Systematic Reviews and Meta-Analyses diagram. PE, patient engagement.

With respect to research design, four studies were qualitative case studies most frequently involving interviews and observation, three employed qualitative interviews and/ or focus groups, and three were cross-sectional surveys. ${ }^{3}$ Two studies collected data from patients, three studies from providers, and five studies from both patients and providers.

\section{Types of PE}

All 10 studies identified types of activities in which patients were engaged for hospital service improvement and their roles in these activities. Little detail was provided about precisely what patients were meant to do or did in relation to these activities. For example, a survey of quality managers identified that in $50.0 \%$ of hospitals, patients were involved in quality improvement projects, and in $64.8 \%$ of hospitals, patients were involved in quality committees, but the survey did not gather specific information about patient involvement in these initiatives such as mode or frequency of engagement, information they contributed or how it was used. ${ }^{20}$ A more informative study involving interviews with nurses and patients on a nurse-led advisory council revealed that patients were involved in a range of activities, including commenting on documents, establishing working groups on specific topics and membership on hospital multidisciplinary groups to conduct service. ${ }^{29}$ Patients were standing members of quality committees, ${ }^{20}$ hospital management committees, ${ }^{22} 28$ or advisory panels, councils or networks. ${ }^{21-232529}$ They were also members of shorter-term project teams. ${ }^{2022} 242527$ In three studies, patients provided solicited feedback by one-time questionnaire or interview about how to improve services. ${ }^{22}{ }^{26}$ In one study, patients voluntarily provided feedback about services by mass media or suggestion boxes. ${ }^{28}$ In one study, patients provided education to other patients. ${ }^{25} \mathrm{~Pa}-$ tients assumed several roles in these activities: developing quality criteria, ${ }^{20}$ reviewing quality improvement project results, ${ }^{20} 29$ identifying issues that warranted improvement, ${ }^{20} 222628$ suggesting potential solutions for addressing problems,${ }^{25}$ and informing the design or reorganisation of services. ${ }^{20} 232427$

Types of PE activities for hospital service improvement were characterised by continuum of engagement (table 1). Three studies (30.0\%) focused on consultation activities: questionnaire, interview, mass media and suggestion boxes. ${ }^{22} 26{ }^{28}$ Eight studies $(80.0 \%)$ focused on involvement activities: members of standing committees, advisory bodies, project teams or providing education to other patients. ${ }^{20-25} 2729$ One study focused on a partnership where citizen advisory panel recommendations on core services were adopted by a hospital board for a restructuring initiative. ${ }^{23}$

\section{Extent of PE}

Five $(50.0 \%)$ studies described the extent to which patients were engaged for hospital service improvement as members of standing committees or project teams. A survey of providers at 74 European hospitals found that patients were infrequently involved in activities such as developing quality criteria or designing or reorganising services (range: $50.0 \%-64.8 \%$ ), and this did not differ across clinical departments. ${ }^{20}$ Observation of 10 hospital committee meetings and 11 community network meetings in eight regions revealed that patient input was minimal; even when they contributed, their influence on decisions was minimal, and their ideas were not pursued by providers subsequent to meetings. ${ }^{21}$ In another study, 
Table 1 Types of patient engagement activities for hospital service improvement ${ }^{9}$

\section{Consultation}

- Provide solicited feedback by questionnaire or interview on how to improve services 2226

- Provide voluntary feedback through mass media or suggestion boxes ${ }^{28}$

\section{Involvement}

- Members of quality committees ${ }^{20}$

- Members of hospital management committees $^{2128}$

- Members of advisory panels, councils or networks ${ }^{21-232529}$

- Members of project teams 2022242527

- Provide education to other patients ${ }^{25}$

\section{Partnership and shared} leadership

Members of a citizen advisory panel that prioritised core services to inform hospital restructuring; recommendations were largely adopted by the hospital board ${ }^{23}$ observation of 21 planning committee meetings across five sites found that patients attended but were largely silent, and both patients and providers agreed patients were not effectively involved in meetings as partners and their suggestions were ignored.$^{24}$ A survey of 142 providers who had been on committees with patients revealed that patients did not often fulfil the roles of sharing their experiences, asking difficult questions or improving communication between the health service and the community. ${ }^{27}$ Interviews with providers, and focus groups with patients and providers revealed that patients did not perceive themselves to be partners in improving service delivery, and hospital management committees or providers did not use patient feedback. ${ }^{28}$

\section{Factors influencing PE}

Seven studies $(70.0 \%)$ assessed facilitators or barriers to $\mathrm{PE}$ for hospital service improvement (table 2). Of the 10 distinct barriers identified, most pertained to providers including negative beliefs and attitudes about patient roles and input, lack of knowledge and skills, hierarchies and dysfunction among providers, and uncertainty about how to resolve differing patient and senior management priorities. Of the 10 distinct facilitators, most pertained

Table 2 Factors influencing patient engagement for hospital service improvement

Facilitators Barriers

Selection of patients based on their personal characteristics and skills ${ }^{24}$

- Involving patients and staff with the desire to work together $^{24}$

- Involving supportive staff with leverage to navigate hospital processes to effect change ${ }^{21}$

- Early involvement in projects so that patients were familiar with objectives and could offer meaningful contributions to shape the project's aims and activities ${ }^{22}$

- Meeting monthly or more frequently if needed ${ }^{24}$

- Small team size that was less hierarchical and more easily integrated patients ${ }^{24}$

- Explicit effort to involve patients in meetings and extend value and respect for their input 222429

- Debriefing with patients after meetings to gather feedback about how the session had gone and how interaction could be improved ${ }^{22}$

- Formal interaction supplemented with informal interaction by email, telephone or other interaction to build relationships ${ }^{22}$

- Formalising patient roles by labelling and recognising their position $^{22} 24$

- Asking patients about feelings to prompt detailed accounts of their experiences ${ }^{26}$

- Joint training of patients and healthcare professionals ${ }^{22}$

- Patient recommendations that align with what healthcare professionals consider appropriate ${ }^{25}$
- Lack of knowledge among healthcare professionals on how to engage with an empowered group of questioning patients ${ }^{29}$

- Lack of guidance on the role of patients and how they should be involved ${ }^{24} 27$

- Healthcare professional beliefs about the relevance and representativeness of individual patient experiences ${ }^{21} 28$

- Healthcare professional beliefs about patient capacity to contribute given lack of criteria for inclusion or a vetting process $^{24}$

- Healthcare professional beliefs that patient feedback was complaining and patients were hostile and ungrateful ${ }^{28} 29$

- Infrequent meetings ${ }^{24}$

- Disagreement between patients and healthcare professionals on the role of patients ${ }^{27}$

- Lack of informal opportunities outside of meetings for interaction to build trust ${ }^{24}$

- Dysfunction and hierarchies among the healthcare professionals $^{24}$

- Pressure from senior management to achieve specific objectives that diverged from patient objectives ${ }^{29}$ 
to processes that optimise PE such as formalising patient roles, small team size, frequent meetings, active solicitation of patient input, and debriefing after meetings.

Three studies (30.0\%) explored the mechanism by which patients influenced the beliefs or actions of providers with whom they interacted. Patient influence appeared to occur through participation in training and meetings with providers, ${ }^{22}$ and outside of formal meetings during opportunistic interaction between patients and providers who were supportive and navigated hospital processes to effect change. ${ }^{21}$ Patient influence was greater when it provided unique insight into problems that allowed providers to identify new possibilities for solutions compared with patients recommending solutions and was viewed as particularly valuable when it aligned with what providers viewed as appropriate. ${ }^{25}$

\section{PE interventions}

No studies developed or evaluated behavioural interventions that would promote or support $\mathrm{PE}$ for hospital service improvement by influencing patient or provider beliefs or actions.

\section{PE impact}

No studies assessed the impact of PE for hospital service improvement on clinical outcomes. A single study evaluated the impact of PE on participants and health services. Observation of five full-day meetings of a 28-member citizen panel convened to establish hospital restructuring priorities and survey of participants after each meeting found they were enthusiastic about the experience and thought sessions were well organised and the facilitators effective, although some patients were anxious about the magnitude and complexity of the task. ${ }^{23}$ Participants thought the panel had accomplished something important that benefited the community and the hospital, and the citizen panel was an effective way to incorporate the community's perspective in decision making. The hospital board approved nearly all panel recommendations resulting in the closure of 26 beds, two outpatient programmes, integration of a programme with the emergency department, and a transition strategy; changes resulted in a balanced budget in both the 2010-2011 and 2011-2012 fiscal years.

\section{DISCUSSION}

This scoping review identified few studies published since 2006 that evaluated PE based on consultation, involvement or partnership for hospital service improvement. No study evaluated interventions that would promote or support PE by influencing patient or provider beliefs or actions. Most studies examined patient involvement in standing committees or projects but few details were provided about these activities. Patient input and influence on decisions was minimal. Only one study evaluated how PE impacted hospital services and no study evaluated the impact of $\mathrm{PE}$ on clinical outcomes. Barriers to
PE were primarily at the provider level including negative beliefs and attitudes about patient roles and input, lack of knowledge and skills, provider dysfunction and hierarchies, and uncertainty about how to resolve differences between patient and senior management priorities. Facilitators of PE included joint training of patients and providers, formalising patient roles, informal interaction to build trust, involving them from the outset of projects, small team size, frequent meetings, active solicitation of patient input during meetings and debriefing after meetings.

The strengths of this study include use of rigorous scoping review methods, ${ }^{14}{ }^{15}$ compliance with standards for the conduct and reporting of reviews, ${ }^{16}$ and use of a framework of patient and family engagement to characterise PE activities. ${ }^{9}$ Several issues may limit the interpretation and application of the findings. Despite having conducted a comprehensive search of multiple databases that complied with standards for search strategies, ${ }^{17}$ it was limited to English language studies. We did not search the grey literature given the methodological challenges that have been identified by others; as a result, important information may have been missed. ${ }^{18} 19$ The search strategy may not have identified all relevant studies or our screening criteria may have been too stringent. Few studies were eligible that provided little specific details about what patients were meant to do or actually did. Risk of bias of included studies was not assessed as this is not customary for a scoping review. Although scoping reviews often include consultation with stakeholders to interpret the findings, ${ }^{15}$ this step was not done because studies were few and provided sparse details.

The findings of this study concur with previous research in primary care, which revealed that $\mathrm{PE}$ for service improvement was token in nature, thus patients did not inform service design or improvement. ${ }^{10-12}$ Barriers (provider beliefs and skills) and facilitators (clearly defined roles for patients, joint training of patients and providers) of PE in the primary care setting also emerged in this study. ${ }^{10-12}$ However, this study identified additional barriers and facilitators that may be unique to the hospital context. Identifying barriers and facilitators is an important first step in the selection and tailoring of behavioural interventions for patients and providers that could be implemented to promote and support PE. ${ }^{30}$ Having identified barriers and facilitators is particularly important given the paucity of included studies that evaluated behavioural interventions for PE.

This review identified two mechanisms underlying the success of PE for hospital service improvement, both of which may mitigate the lack of skills and negative beliefs among providers that were consistent barriers to PE. These mechanisms should be considered when designing $\mathrm{PE}$ activities. One included study found that patient influence on hospital services was more likely when providers were supportive and navigated hospital processes to effect change. ${ }^{21}$ It may be crucial to carefully select and/or train providers who participate in committees or project 
teams with patients so that they function as champions. It is well recognised that champions can improve service delivery and clinical outcomes ${ }^{31}$ by influencing the knowledge and behaviour of their colleagues. ${ }^{32}$ Analysis of findings reported in included studies that pertain to providers offers additional insight into potential ways to prepare and support providers so that they can, in turn, support patients in their PE role. For example, provider-level barriers to PE (eg, lack of knowledge of how to engage patients, attitudes about PE, hierarchies and dysfunction among providers) suggest that providers require $\mathrm{PE}$ training, and senior leaders must ensure that a culture of $\mathrm{PE}$ is implemented including infrastructure and processes. Another included study found that patient influence on hospital services was more likely when patients were asked to provide unique insight into problems that allowed providers to identify new possibilities for solutions rather than for patients to recommend solutions that were not viewed by providers as relevant or appropriate. ${ }^{25}$ This approach could be accommodated in PE activities that consulted, involved or partnered with patients, appeared to be a comfortable role that allowed patients to express themselves freely and provide detailed accounts of their experiences, ${ }^{26}$ and would ensure that providers were engaged rather than alienated by the process.

The value of a scoping review is to reveal issues for which knowledge is lacking and warrants future research. This study identified several such issues. Few types of PE activities for hospital service improvement were examined in included studies. Therefore, ongoing primary research should develop and evaluate the same and additional types of PE activities. These activities should address the continuum of PE including consultation, involvement and partnership, since all three categories of PE activities may be appropriate for different health service improvement objectives. This review found that patient input and influence on decisions was minimal. Therefore, further primary research is needed to develop and evaluate behavioural interventions that support PE for hospital service improvement directed at patients and providers informed by the PE barriers and facilitators identified here. Matching of barriers and facilitators to relevant interventions could be informed by taxonomies of behaviour change interventions such as the Expert Recommendations for Implementing Change, ${ }^{33}$ or the Effective Practice and Organisation of Care taxonomy, ${ }^{34}$ and formal processes for developing behavioural interventions such as Intervention Mapping. ${ }^{35}$ Instruments exist to assess patient readiness for $\mathrm{PE}$, for example, the patient activation measure; these could be employed to evaluate the impact of behavioural interventions used to prepare patients for PE. ${ }^{36}$ One study only examined the impact of $\mathrm{PE}$ on participants and health services; none assessed the impact of PE on clinical outcomes. Thus, ongoing research that tests the effectiveness of $\mathrm{PE}$ behavioural interventions should assess the impact on various individual and organisational outcomes.

\section{CONCLUSIONS}

Few studies have evaluated PE for hospital service improvement to identify the best activities, roles and behavioural interventions for patients and providers that support PE and result in improved health services and patient outcomes. Lack of skill and negative beliefs among providers were a consistent barrier to PE. This review identified numerous facilitators and mechanisms that could be employed by hospitals to optimise PE for service improvement and its impact. Further research is needed to elaborate on PE activities suitable for consultation, involvement and partnership; test behavioural interventions for PE directed at patients and providers informed by the PE barriers and facilitators identified here; and demonstrate the impact of PE. This would identify types of PE activities and supportive conditions that should be prioritised by hospitals.

Contributors ARG envisioned and planned the study, and provided funding for research assistant support. LL, AC, RU, SES, WPW, RB and ARG established study objectives, collected and analysed or interpreted data, drafted or edited the manuscript, and read and approved the final manuscript.

Funding This research received no specific grant from any funding agency in the public, commercial or not-for-profit sectors.

Competing interests None declared.

Patient consent Not required.

Provenance and peer review Not commissioned; externally peer reviewed.

Data sharing statement All data are available in the manuscript or supplementary files.

Open Access This is an Open Access article distributed in accordance with the Creative Commons Attribution Non Commercial (CC BY-NC 4.0) license, which permits others to distribute, remix, adapt, build upon this work non-commercially, and license their derivative works on different terms, provided the original work is properly cited and the use is non-commercial. See: http://creativecommons.org/ licenses/by-nc/4.0/

(c) Article author(s) (or their employer(s) unless otherwise stated in the text of the article) 2018. All rights reserved. No commercial use is permitted unless otherwise expressly granted.

\section{REFERENCES}

1. Berwick DM. Era 3 for medicine and health care. JAMA 2016;315:1329-30.

2. Rathert C, Wyrwich MD, Boren SA. Patient-centered care and outcomes: a systematic review of the literature. Med Care Res Rev 2013;70:351-79.

3. Coulter A, Ellins J. Effectiveness of strategies for informing, educating, and involving patients. BMJ 2007;335:24-7.

4. Mead N, Bower P. Patient-centredness: a conceptual framework and review of the empirical literature. Soc Sci Med 2000;51:1087-110.

5. Mockford C, Staniszewska S, Griffiths F, et al. The impact of patient and public involvement on UK NHS health care: a systematic review. Int J Qual Health Care 2012;24:28-38.

6. Prey JE, Woollen J, Wilcox L, et al. Patient engagement in the inpatient setting: a systematic review. J Am Med Inform Assoc 2014;21:742-50.

7. Constand MK, MacDermid JC, Dal Bello-Haas V, et al. Scoping review of patient-centered care approaches in healthcare. BMC Health Serv Res 2014;14:271.

8. Berger Z, Flickinger TE, Pfoh E, et al. Promoting engagement by patients and families to reduce adverse events in acute care settings: a systematic review. BMJ Qual Saf 2014;23:548-55.

9. Carman KL, Workman TA. Engaging patients and consumers in research evidence: applying the conceptual model of patient and family engagement. Patient Educ Couns 2017;100:25-9. 
10. Baker GR, Fancott C, Judd M, et al. Expanding patient engagement in quality improvement and health system redesign: three Canadian case studies. Healthc Manage Forum 2016;29:176-82.

11. Johnson KE, Mroz TM, Abraham M, et al. Promoting patient and family partnerships in ambulatory care improvement: a narrative review and focus group findings. Adv Ther 2016;33:1417-39.

12. van $\mathrm{C}$, Mclnerney $\mathrm{P}$, Cooke R. Patients' involvement in improvement initiatives: a qualitative systematic review. JBI Database System Rev Implement Rep 2015;13:232-90.

13. Aboumatar $\mathrm{HJ}$, Chang $\mathrm{BH}, \mathrm{Al}$ Danaf J, et al. Promising practices for achieving patient-centered hospital care: a national study of highperforming US Hospitals. Med Care 2015;53:758-67.

14. Arksey H, O'Malley L. Scoping studies: towards a methodological framework. Int J Soc Res Methodol 2005;8:19-32.

15. Levac D, Colquhoun H, O'Brien KK. Scoping studies: advancing the methodology. Implement Sci 2010;5:69.

16. Moher D, Liberati A, Tetzlaff J, et al. Preferred reporting items for systematic reviews and meta-analyses: the PRISMA statement. PLoS Med 2009;6:e1000097.

17. McGowan J, Sampson M, Salzwedel DM, et al. PRESS Peer Review of Electronic Search Strategies: 2015 guideline statement. J Clin Epidemiol 2016;75:40-6.

18. Benzies KM, Premji S, Hayden KA, et al. State-of-the-evidence reviews: advantages and challenges of including grey literature. Worldviews Evid Based Nurs 2006;3:55-61.

19. Adams J, Hillier-Brown FC, Moore HJ, et al. Searching and synthesising 'grey literature' and 'grey information' in public health: critical reflections on three case studies. Syst Rev 2016;5:164.

20. Groene O, Sunol R, Klazinga NS, et al. Involvement of patients or their representatives in quality management functions in $\mathrm{EU}$ hospitals: implementation and impact on patient-centred care strategies. Int J Qual Health Care 2014;26:81-91.

21. Nathan S, Stephenson N, Braithwaite J. Sidestepping questions of legitimacy: how community representatives manoeuvre to effect change in a health service. Health 2014;18:23-40.

22. Armstrong N, Herbert G, Aveling EL, et al. Optimizing patient involvement in quality improvement. Health Expect 2013;16:e36-47.

23. Chan YE, Benecki LA. Evaluating the success of a hospital's community engagement process. Healthc Manage Forum 2013;26:20-5.
24. Martin GP, Finn R. Patients as team members: opportunities, challenges and paradoxes of including patients in multi-professional healthcare teams. Sociol Health IIIn 2011;33:1050-65.

25. Solbjør M, Steinsbekk A. User involvement in hospital wards: professionals negotiating user knowledge. A qualitative study. Patient Educ Couns 2011;85:e144-149.

26. Hsu MY, McCormack B. The experience of applying a narrative research approach with older people. J Nurs Res 2010;18:249-57.

27. Nathan S, Johnston L, Braithwaite J. The role of community representatives on health service committees: staff expectations vs. reality. Health Expect 2011;14:272-84.

28. Rutebemberwa E, Ekirapa-Kiracho E, Okui O, et al. Lack of effective communication between communities and hospitals in Uganda: a qualitative exploration of missing links. BMC Health Serv Res 2009;9:146

29. Brooks F. Nursing and public participation in health: an ethnographic study of a patient council. Int J Nurs Stud 2008;45:3-13.

30. Krause J, Van Lieshout J, Klomp R, et al. Identifying determinants of care for tailoring implementation in chronic diseases: an evaluation of different methods. Implement Sci 2014;9:102.

31. Flodgren G, Parmelli E, Doumit G, et al. Local opinion leaders: effects on professional practice and health care outcomes. Cochrane Database Syst Rev 2011;8:CD000125.

32. Gabbay J, le May A. Evidence based guidelines or collectively constructed "mindlines?" Ethnographic study of knowledge management in primary care. BMJ 2004;329:1013.

33. Powell BJ, Waltz TJ, Chinman MJ, et al. A refined compilation of implementation strategies: results from the Expert Recommendations for Implementing Change (ERIC) project. Implement Sci 2015;10:21.

34. Effective Practice and Organisation of Care (EPOC). EPOC taxonomy. 2015 https://epoc.cochrane.org/epoc-taxonomy

35. Bartholomew LK, Parcel GS, Kok G. Intervention mapping: a process for developing theory- and evidence-based health education programs. Health Educ Behav 1998;25:545-63.

36. Hibbard JH, Stockard J, Mahoney ER, et al. Development of the Patient Activation Measure (PAM): conceptualizing and measuring activation in patients and consumers. Health Serv Res 2004;39:1005-26. 